\title{
Richardson extrapolation for the discrete iterated modified projection solution
}

\author{
Rekha P. KULKARNI *and Gobinda RAKSHIT ${ }^{\dagger}$
}

\begin{abstract}
Approximate solutions of Urysohn integral equations using projection methods involve integrals which need to be evaluated using a numerical quadrature formula. It gives rise to the discrete versions of the projection methods. For $r \geq 1$, a space of piecewise polynomials of degree $\leq r-1$ with respect to an uniform partition is chosen to be the approximating space and the projection is chosen to be the interpolatory projection at $r$ Gauss points. Asymptotic expansion for the iterated modified projection solution is available in literature. In this paper, we obtain an asymptotic expansion for the discrete iterated modified projection solution and use Richardson extrapolation to improve the order of convergence. Our results indicate a choice of a numerical quadrature which preserves the order of convergence in the continuous case. Numerical results are given for a specific example.
\end{abstract}

Key Words : Urysohn integral operator, Interpolatory projection, Gauss points, Nyström Approximation, Richardson extrapolation.

AMS subject classification : 45G10, 65J15, 65R20

*Department of Mathematics, I.I.T. Bombay, Powai, Mumbai 400076, India, rpk@math.iitb.ac.in,

${ }^{\dagger}$ gobindarakshit@math.iitb.ac.in 


\section{Introduction}

Let $\mathscr{X}=L^{\infty}[0,1]$ and consider the following Urysohn integral operator

$$
\mathscr{K}(x)(s)=\int_{0}^{1} \kappa(s, t, x(t)) d t, \quad s \in[0,1], x \in \mathscr{X},
$$

where $\kappa(s, t, u)$ is a continuous real valued function defined on $\Omega=[0,1] \times[0,1] \times \mathbb{R}$. Then $\mathscr{K}$ is a compact operator from $L^{\infty}[0,1]$ to $C[0,1]$. Assume that for $f \in C[0,1]$,

$$
x-\mathscr{K}(x)=f
$$

has a unique solution $\varphi$.

We are interested in computable approximate solutions of the above equation. We consider some projection methods associated with a sequence of interpolatory projections converging to the Identity operator pointwise.

For $r \geq 1$, let $\mathscr{X}_{n}$ denote the space of piecewise polynomials of degree $\leq r-1$ with respect

to a uniform partition of $[0,1]$ with $n$ subintervals. Let $h=\frac{1}{n}$ denote the mesh of the partition and let $Q_{n}: C[0,1] \rightarrow \mathscr{X}_{n}$ be the interpolation operator at $r$ Gauss points. In Grammont et al [5] the following modified projection method is investigated:

$$
\varphi_{n}^{M}-\mathscr{K}_{n}^{M}\left(\phi_{n}^{M}\right)=f
$$

where

$$
\mathscr{K}_{n}^{M}(x)=Q_{n} \mathscr{K}(x)+\mathscr{K}\left(Q_{n} x\right)-Q_{n} \mathscr{K}\left(Q_{n} x\right)
$$

The iterated modified projection solution is defined as

$$
\tilde{\varphi}_{n}^{M}=\mathscr{K}\left(\phi_{n}^{M}\right)+f
$$

If $\frac{\partial \kappa}{\partial u} \in C^{3 r}(\Omega)$ and $f \in C^{2 r}[0,1]$, then the following orders of convergence are proved in Grammont et al [5]:

$$
\left\|\varphi-\varphi_{n}^{M}\right\|_{\infty}=O\left(h^{3 r}\right), \quad\left\|\varphi-\tilde{\varphi}_{n}^{M}\right\|_{\infty}=O\left(h^{4 r}\right) .
$$

Under the assumption of $\frac{\partial \kappa}{\partial u} \in C^{3 r+3}(\Omega)$ and $f \in C^{2 r+2}[0,1]$, the following asymptotic series expansion is obtained in Kulkarni-Nidhin [6] :

$$
\tilde{\varphi}_{n}^{M}=\varphi+\zeta h^{4 r}+O\left(h^{4 r+2}\right)
$$


where the function $\zeta$ is independent of $h$.

In practice, it is necessary to replace all the integrals by a numerical quadrature formula, giving rise to the discrete versions of the above methods.

We choose a composite numerical quadrature formula with a degree of precision $d-1$ and with respect to a uniform partition of $[0,1]$ with $m=n p, p \in \mathbb{N}$, subintervals. Let $\tilde{h}=\frac{1}{m}$ denote the mesh of this fine partition. Then the Nyström operator $\mathscr{K}_{m}$ is obtained by replacing the integral in the definition (1.1) of $\mathscr{K}$ and the discrete version of the operator $\mathscr{K}_{n}^{M}$ defined in (1.3) is obtained on replacing $\mathscr{K}$ by $\mathscr{K}_{m}$ and is denoted by $\tilde{\mathscr{K}}_{n}^{M}$. The Nyström solution is denoted by $\varphi_{m}$ and it satisfies

$$
\varphi_{m}-\mathscr{K}_{m}\left(\varphi_{m}\right)=f
$$

The discrete modified projection and the discrete iterated modified projection solutions are denoted respectively by $z_{n}^{M}$ and $\tilde{z}_{n}^{M}$ and are defined as

$$
z_{n}^{M}-\tilde{\mathscr{K}}_{n}^{M}\left(z_{n}^{M}\right)=f, \quad \tilde{z}_{n}^{M}=\mathscr{K}_{m} z_{n}^{M}+f
$$

In Kulkarni-Rakshit [7] the following estimates are proved:

$$
\begin{aligned}
& \text { If } d \geq 2 r, \kappa \in C^{d}(\Omega), \quad \frac{\partial \kappa}{\partial u} \in C^{2 r}(\Omega) \text { and } f \in C^{d}([0,1]) \text {, then } \\
& \left\|z_{n}^{M}-\varphi\right\|_{\infty}=O\left(\max \left\{\tilde{h}^{d}, h^{3 r}\right\}\right) . \\
& \qquad \begin{array}{r}
\text { If } d \geq 2 r, \quad \frac{\partial \kappa}{\partial u} \in C^{\max \{d, 3 r\}}(\Omega) \text { and } f \in C^{d}([0,1]), \text { then } \\
\|\varphi\|_{\infty}=O\left(\max \left\{\tilde{h}^{d}, h^{4 r}\right\}\right) .
\end{array}
\end{aligned}
$$

In this paper we show that if

$$
d \geq 2 r, \frac{\partial \kappa}{\partial u} \in C^{\max \{d, 3 r+3\}}(\Omega), \quad \text { and } \quad f \in C^{\max \{d, 2 r+2\}}[0,1]
$$

then in fact

$$
\tilde{z}_{n}^{M}=\varphi_{m}+\alpha h^{4 r}+O\left(h^{2 r} \max \left\{\tilde{h}^{d}, h^{2 r+2}\right\}\right)
$$

where the term $\alpha$ is independent of $h$. If we choose $\tilde{h}$ and $d$ such that $\tilde{h}^{d} \leq h^{2 r+2}$, then using the Richardson extrapolation, an estimate of $\varphi_{m}$ of the order of $h^{4 r+2}$ could be obtained. 
The proof of (1.4) is based on an asymptotic expansion for the term $\mathscr{K}^{\prime}(\varphi)\left(Q_{n} v-v\right)$, where $\mathscr{K}^{\prime}(\varphi)$ denotes the Fréchet derivative of $\mathscr{K}$ at $\varphi$ and $v$ is a smooth function. This is obtained by using the Euler-MacLaurin formula. The main difficulty in proving (1.7) is that a discrete version of the Euler-MacLaurin formula is not available which is needed for obtaining an asymptotic expansion for $\mathscr{K}_{m}^{\prime}\left(\varphi_{m}\right)\left(Q_{n} v-v\right)$. We prove (1.7) using a different approach.

The paper is arranged as follows. In Section 2 we define discrete versions of the modified projection method and of its iterated version. Section 3 is devoted to the asymptotic series expansions for $\mathscr{K}_{m}^{\prime}\left(\varphi_{m}\right)\left(Q_{n} v-v\right)$ and for terms involving higher order Fréchet derivatives of the operator $\mathscr{K}_{m}$ at $\varphi_{m}$. In Section 4 we prove our main result, that is, the expansion (1.7). Numerical results are given in Section 5.

\section{Discrete Projection Methods}

In the discrete projection methods, all the integrals are replaced by a numerical quadrature formula. We first define a numerical quadrature formula and the Nyström operator $\mathscr{K}_{m}$ which approximates $\mathscr{K}$. Let $m \in \mathbb{N}$ and consider the following uniform partition of $[0,1]$ :

$$
\Delta_{m}: 0<\frac{1}{m}<\cdots<\frac{m-1}{m}<1 .
$$

Let

$$
\tilde{h}=\frac{1}{m} \quad \text { and } \quad s_{i}=\frac{i}{m}, \quad i=0, \ldots, m .
$$

Consider a basic quadrature rule

$$
\int_{0}^{1} f(t) d t \approx \sum_{i=1}^{\rho} \omega_{i} f\left(\mu_{i}\right)
$$

and let

$$
\zeta_{i}^{j}=s_{j-1}+\mu_{i} \tilde{h}, \quad i=1, \ldots, \rho, \quad j=1, \ldots, m .
$$

A composite integration rule with respect to the partition (2.1) is then defined as

$$
\int_{0}^{1} f(t) d t=\sum_{j=1}^{m} \int_{s_{j-1}}^{s_{j}} f(t) d t \approx \tilde{h} \sum_{j=1}^{m} \sum_{i=1}^{\rho} \omega_{i} f\left(\zeta_{i}^{j}\right) .
$$

For $k \geq 1$, let

$$
C_{\Delta_{m}}^{k}([0,1])=\left\{g \in L^{\infty}[0,1]:\left.g\right|_{\left[s_{j-1}, s_{j}\right]} \in C^{k}\left(\left[s_{j-1}, s_{j}\right]\right), j=1, \ldots, m\right\} .
$$


The error in the numerical quadrature is assumed to be of the following form: There is a positive integer $d$ such that if $f \in C_{\Delta_{m}}^{d}([0,1])$, then

$$
\left|\int_{0}^{1} f(t) d t-\tilde{h} \sum_{j=1}^{m} \sum_{i=1}^{\rho} \omega_{i} f\left(\zeta_{i}^{j}\right)\right| \leq C_{1}\left\|f^{(d)}\right\|_{\infty} \tilde{h}^{d}
$$

where $f^{(d)}$ denotes $d$ th derivative of $f$ and $C_{1}$ is a constant independent of $\tilde{h}$.

Assume that $\kappa \in C^{d}(\Omega)$ and that $f \in C^{d}([0,1])$. Then it follows that $\varphi \in C^{d}([0,1])$. We also assume throughout that $\left(I-\mathscr{K}^{\prime}(\varphi)\right)^{-1}$ is a bounded linear operator from $C[0,1]$ to itself.

We replace the integral in (1.1) by the numerical quadrature formula (2.2) and define the Nyström operator as

$$
\mathscr{K}_{m}(x)(s)=\tilde{h} \sum_{j=1}^{m} \sum_{i=1}^{\rho} \omega_{i} \kappa\left(s, \zeta_{i}^{j}, x\left(\zeta_{i}^{j}\right)\right), \quad s \in[0,1], \quad x \in C([0,1]) .
$$

Then since $\kappa \in C^{d}(\Omega)$ and $\varphi \in C^{d}([0,1])$, it follows that

$$
\left\|\mathscr{K}(\varphi)-\mathscr{K}_{m}(\varphi)\right\|_{\infty}=O\left(\tilde{h}^{d}\right)
$$

In the Nyström method, (1.2) is approximated by

$$
x_{m}-\mathscr{K}_{m}\left(x_{m}\right)=f .
$$

Fix $\delta>0$ and define

$$
B(\varphi, \delta)=\left\{x:\|x-\varphi\|_{\infty} \leq \delta\right\}
$$

Then for $m$ sufficiently large, the equation (2.5) has a unique solution $\varphi_{m}$ in $B(\varphi, \delta)$ and

$$
\left\|\varphi-\varphi_{m}\right\|_{\infty}=O\left(\tilde{h}^{d}\right)
$$

Note that the Fréchet derivative of $\mathscr{K}_{m}$ is given by

$$
\mathscr{K}_{m}^{\prime}(x) v(s)=\tilde{h} \sum_{j=1}^{m} \sum_{i=1}^{\rho} \omega_{i} \frac{\partial \kappa}{\partial u}\left(s, \zeta_{i}^{j}, x\left(\zeta_{i}^{j}\right)\right) v\left(\zeta_{i}^{j}\right), \quad s \in[0,1], \quad v \in C([0,1]) .
$$

If $\frac{\partial \kappa}{\partial u} \in C^{d}(\Omega)$ and $v \in C_{\Delta_{m}}^{d}([0,1])$, then from (2.3)

$$
\left\|\mathscr{K}^{\prime}(\varphi) v-\mathscr{K}_{m}^{\prime}(\varphi) v\right\|_{\infty} \leq C_{2}\|v\|_{d, \infty} \tilde{h}^{d}
$$


where $\|v\|_{d, \infty}=\max _{0 \leq j \leq d}\left\|v^{(j)}\right\|_{\infty}$ and $C_{2}$ is a constant independent of $\tilde{h}$.

We now define the interpolatory projection at $r$ Gauss points. Let $n \in \mathbb{N}$ and consider the following uniform partition of $[0,1]$ :

$$
\Delta_{n}: 0<\frac{1}{n}<\cdots<\frac{n-1}{n}<1 .
$$

Define

$$
t_{j}=\frac{j}{n}, \quad j=0, \ldots, n \text { and } h=t_{j}-t_{j-1}=\frac{1}{n} .
$$

For a positive integer $r$, let

$$
\mathscr{X}_{n}=\left\{g \in L^{\infty}[0,1]:\left.g\right|_{\left[t_{j-1}, t_{j}\right]} \text { is a polynomial of degree } \leq r-1, j=1, \ldots, n\right\} .
$$

Let $\left\{q_{1}, \cdots, q_{r}\right\}$ denote the Gauss-Legendre zeros of order $r$ in $[0,1]$. The $n r$ collocation nodes are chosen as follows:

$$
\tau_{i}^{k}=t_{k-1}+q_{i} h, \quad i=1, \ldots, r, \quad k=1, \ldots, n .
$$

Define the interpolation operator $Q_{n}: C[0,1] \rightarrow \mathscr{X}_{n}$ as

$$
\left(Q_{n} x\right)\left(\tau_{i}^{k}\right)=x\left(\tau_{i}^{k}\right), \quad i=1, \ldots, r, \quad k=1, \ldots, n
$$

Using the Hahn-Banach extension theorem as in Atkinson et al [3], $Q_{n}$ can be extended to $L^{\infty}[0,1]$. Note that for $x \in C[0,1], Q_{n} x \rightarrow x$ as $n \rightarrow \infty$. As a consequence,

$$
\sup _{n}\left\|\left.Q_{n}\right|_{C[0,1]}\right\| \leq C_{3}
$$

Also, if $u \in C^{r}([0,1])$, then

$$
\left\|u-Q_{n} u\right\|_{\infty} \leq C_{4}\left\|u^{(r)}\right\|_{\infty} h^{r}
$$

where $C_{4}$ is a constant independent of $n$. Throughout this paper we choose $d \geq 2 r$ and $m=p n$ for some $p \in \mathbb{N}$. Then $\tilde{h}=\frac{h}{p} \leq h$. It follows from (2.6), (2.8) and (2.9) that

$$
\left\|\varphi_{m}-Q_{n} \varphi_{m}\right\|_{\infty} \leq\left\|\varphi-Q_{n} \varphi\right\|_{\infty}+\left\|\left(I-Q_{n}\right)\left(\varphi_{m}-\varphi\right)\right\|_{\infty}=O\left(\max \left\{h^{r}, \tilde{h}^{d}\right\}\right) .
$$

Hence it follows that

$$
\left\|\varphi_{m}-Q_{n} \varphi_{m}\right\|_{\infty}=O\left(h^{r}\right)
$$


The discrete modified projection operator is defined by replacing $\mathscr{K}$ with $\mathscr{K}_{m}$ in (1.3) as follows:

$$
\tilde{\mathscr{K}}_{n}^{M}(x)=Q_{n} \mathscr{K}_{m}(x)+\mathscr{K}_{m}\left(Q_{n} x\right)-Q_{n} \mathscr{K}_{m}\left(Q_{n} x\right)
$$

Discrete Modified Projection Method: For $n$ and $m$ big enough,

$$
x_{n}-\tilde{\mathscr{K}}_{n}^{M}\left(x_{n}\right)=f
$$

has a unique solution $z_{n}^{M}$ in a $B(\varphi, \delta)$.

The Discrete Iterated Modified Projection solution is defined as

$$
\tilde{z}_{n}^{M}=\mathscr{K}_{m}\left(z_{n}^{M}\right)+f
$$

\section{$3 \quad$ Asymptotic Series Expansions}

By assumption, $I-\mathscr{K}^{\prime}(\varphi)$ is invertible. Let

$$
M=\left(I-\mathscr{K}^{\prime}(\varphi)\right)^{-1} \mathscr{K}^{\prime}(\varphi), \quad \Psi(t)=\left(t-q_{1}\right) \cdots\left(t-q_{r}\right), t \in[0,1] .
$$

We quote the following asymptotic series expansions from Kulkarni-Nidhin [6].

If $\frac{\partial \kappa}{\partial u} \in C^{2 r+2}(\Omega)$ and $\psi \in C^{2 r+2}([0,1])$, then

$$
\begin{gathered}
\mathscr{K}^{\prime}(\varphi)\left(Q_{n} \psi-\psi\right)=T(\psi) h^{2 r}+O\left(h^{2 r+2}\right), \\
M\left(Q_{n} \psi-\psi\right)=U(\psi) h^{2 r}+O\left(h^{2 r+2}\right),
\end{gathered}
$$

where for $s \in[0,1]$,

$$
\begin{gathered}
T(\psi)(s)=d_{2 r, 2 r} \mathscr{K}^{\prime}(\varphi) \psi^{(2 r)}(s)+\sum_{i=r}^{2 r-1} d_{2 r, i}\left[\left(\frac{\partial}{\partial t}\right)^{2 r-1-i} \ell(s, t) \psi^{(i)}(t)\right]_{t=0}^{1}, \\
U(\psi)(s)=d_{2 r, 2 r} M \psi^{(2 r)}(s)+\sum_{i=r}^{2 r-1} d_{2 r, i}\left[\left(\frac{\partial}{\partial t}\right)^{2 r-1-i} m(s, t) \psi^{(i)}(t)\right]_{t=0}^{1}, \\
d_{2 r, i}=-\int_{0}^{1} \Phi_{i}(\tau) \frac{B_{2 r-i}(\tau)}{(2 r-i) !} \Psi(\tau) d \tau, \quad i=r, \ldots, 2 r
\end{gathered}
$$

with

$$
\Phi_{i}(\tau)=\int_{0}^{1} \frac{(\sigma-\tau)^{i-r}}{(i-r) !} \frac{\left[q_{1}, q_{2}, \cdots, q_{r}, \tau\right](\cdot-\sigma)_{+}^{r-1}}{(r-1) !} d \sigma
$$


Note that the coefficients $d_{2 r, i}$ are independant of $h$. Also,

$$
\mathscr{K}^{\prime \prime}(\varphi)\left(Q_{n} \psi-\psi\right)^{2}=V_{1}(\psi) h^{2 r}+O\left(h^{2 r+2}\right), \quad \mathscr{K}^{(3)}(\varphi)\left(Q_{n} \psi-\psi\right)^{3}=V_{2}(\psi) h^{3 r}+O\left(h^{3 r+1}\right),
$$

where

$$
V_{1}(\psi)=\left(\int_{0}^{1} \Psi(\tau)^{2} \Phi_{r}(\tau)^{2} d \tau\right) \mathscr{K}^{\prime \prime}(\varphi)\left(\psi^{(r)}\right)^{2}
$$

and

$$
V_{2}(\psi)=\left(\int_{0}^{1} \Psi(\tau)^{3} \Phi_{r}(\tau)^{3} d \tau\right) \mathscr{K}^{(3)}(\varphi)\left(\psi^{(r)}\right)^{3}
$$

Let $\frac{\partial^{4} \kappa}{\partial u^{4}} \in C^{2 r}(\Omega)$ and $v_{k} \in C([0,1]), 1 \leq k \leq 4$. The Fréchet derivatives of $\mathscr{K}_{m}$ are as follows:

$$
\mathscr{K}_{m}^{(k)}(x)\left(v_{1}, \ldots, v_{k}\right)(s)=\tilde{h} \sum_{j=1}^{m} \sum_{i=1}^{\rho} \omega_{i} \frac{\partial^{k} \kappa}{\partial u^{k}}\left(s, \zeta_{i}^{j}, x\left(\zeta_{i}^{j}\right)\right) v_{1}\left(\zeta_{i}^{j}\right) \cdots v_{k}\left(\zeta_{i}^{j}\right), \quad s \in[0,1] .
$$

We introduce the following notations:

$$
D^{(i, j, k)} \kappa(s, t, u)=\frac{\partial^{i+j+k} \kappa}{\partial s^{i} \partial t^{j} \partial u^{k}}(s, t, u), \quad C_{5}=\max _{\substack{0 \leq i+j \leq 2 r \\ 1 \leq k \leq 4}} \max _{\substack{s, t \in[0,1] \\ \mid \leq\|\| \varphi \|_{\infty}+\delta}}\left|D^{(i, j, k)} \kappa(s, t, u)\right| .
$$

Using the Mean Value Theorem, it can be seen that

$$
\left\|\mathscr{K}_{m}^{\prime}(\varphi)-\mathscr{K}_{m}^{\prime}\left(\varphi_{m}\right)\right\| \leq\left(\sum_{i=1}^{\rho}\left|\omega_{i}\right|\right) C_{5} \tilde{h}^{d}
$$

We now prove asymptotic series expansions for the first three Fréchet derivatives of the Nyström operator $\mathscr{K}_{m}$ at $\varphi_{m}$.

Proposition 3.1. If $\frac{\partial \kappa}{\partial u} \in C^{\max \{d, 2 r+2\}}(\Omega)$ and $f \in C^{\max \{d, 2 r+2\}}([0,1])$, then

$$
\begin{aligned}
\mathscr{K}_{m}^{\prime}\left(\varphi_{m}\right)\left(Q_{n} \varphi_{m}-\varphi_{m}\right) & =T(\varphi) h^{2 r}+O\left(\max \left\{\tilde{h}^{d}, h^{2 r+2}\right\}\right), \\
\mathscr{K}_{m}^{\prime \prime}\left(\varphi_{m}\right)\left(Q_{n} \varphi_{m}-\varphi_{m}\right)^{2} & =V_{1}(\varphi) h^{2 r}+O\left(\max \left\{\tilde{h}^{d}, h^{2 r+2}\right\}\right), \\
\mathscr{K}_{m}^{(3)}\left(\varphi_{m}\right)\left(Q_{n} \varphi_{m}-\varphi_{m}\right)^{3} & =V_{2}(\varphi) h^{3 r}+O\left(\max \left\{\tilde{h}^{d}, h^{3 r+1}\right\}\right) .
\end{aligned}
$$


Proof. We write

$$
\mathscr{K}_{m}^{\prime}(\varphi)\left(Q_{n} \varphi-\varphi\right)=\mathscr{K}^{\prime}(\varphi)\left(Q_{n} \varphi-\varphi\right)+\left(\mathscr{K}_{m}^{\prime}(\varphi)-\mathscr{K}^{\prime}(\varphi)\right)\left(Q_{n} \varphi-\varphi\right) .
$$

Since $d \geq 2 r$ and $\tilde{h} \leq h$, from the estimate (2.7) we obtain,

$$
\left\|\left(\mathscr{K}_{m}^{\prime}(\varphi)-\mathscr{K}^{\prime}(\varphi)\right)\left(Q_{n} \varphi-\varphi\right)\right\|_{\infty} \leq C_{2}\left\|Q_{n} \varphi-\varphi\right\|_{d, \infty} \tilde{h}^{d}=C_{2}\|\varphi\|_{d, \infty} \tilde{h}^{d} .
$$

Since $\varphi \in C^{2 r+2}[0,1]$, it follows from (3.1) that

$$
\mathscr{K}_{m}^{\prime}(\varphi)\left(Q_{n} \varphi-\varphi\right)=T(\varphi) h^{2 r}+O\left(h^{2 r+2}\right) .
$$

Since by (2.6), $\left\|\varphi-\varphi_{m}\right\|_{\infty}=O\left(\tilde{h}^{d}\right)$, we obtain

$$
\begin{aligned}
\mathscr{K}_{m}^{\prime}(\varphi)\left(Q_{n} \varphi_{m}-\varphi_{m}\right) & =\mathscr{K}_{m}^{\prime}(\varphi)\left(Q_{n} \varphi-\varphi\right)+\mathscr{K}_{m}^{\prime}(\varphi)\left(Q_{n}-I\right)\left(\varphi_{m}-\varphi\right) \\
& =T(\varphi) h^{2 r}+O\left(\max \left\{\tilde{h}^{d}, h^{2 r+2}\right\}\right) .
\end{aligned}
$$

Using the estimate (3.3), we thus obtain

$$
\begin{aligned}
\mathscr{K}_{m}^{\prime}\left(\varphi_{m}\right)\left(Q_{n} \varphi_{m}-\varphi_{m}\right) & =\mathscr{K}_{m}^{\prime}(\varphi)\left(Q_{n} \varphi_{m}-\varphi_{m}\right)+\left[\mathscr{K}_{m}^{\prime}\left(\varphi_{m}\right)-\mathscr{K}_{m}^{\prime}(\varphi)\right]\left(Q_{n} \varphi_{m}-\varphi_{m}\right) \\
& =T(\varphi) h^{2 r}+O\left(\max \left\{\tilde{h}^{d}, h^{2 r+2}\right\}\right) .
\end{aligned}
$$

This completes the proof of (3.4). The proofs of $(\underline{3.5})$ and $(3.6)$ are similar.

\section{Discrete Iterated Modified Projection method}

In this section we prove our main result about the asymptotic series expansion for the discrete iterated modified projection solution $\tilde{z}_{n}^{M}$.

Using the generalised Taylor series expansion, we obtain

$$
\tilde{z}_{n}^{M}-\varphi_{m}=\mathscr{K}_{m}\left(z_{n}^{M}\right)-\mathscr{K}_{m}\left(\varphi_{m}\right)=\mathscr{K}_{m}^{\prime}\left(\varphi_{m}\right)\left(z_{n}^{M}-\varphi_{m}\right)+R\left(z_{n}^{M}-\varphi_{m}\right)
$$

where

$$
R\left(z_{n}^{M}-\varphi_{m}\right)(s)=\int_{0}^{1}(1-\theta) \mathscr{K}_{m}^{\prime \prime}\left(\varphi_{m}+\theta\left(z_{n}^{M}-\varphi_{m}\right)\right)\left(z_{n}^{M}-\varphi_{m}\right)^{2}(s) d \theta
$$


It follows that

$$
\left\|R\left(z_{n}^{M}-\varphi_{m}\right)\right\|_{\infty} \leq \frac{C_{5}}{2}\left(\sum_{i=1}^{\rho}\left|\omega_{i}\right|\right)\left\|z_{n}^{M}-\varphi_{m}\right\|_{\infty}^{2}
$$

If $d \geq 2 r, \kappa \in C^{d}(\Omega), \frac{\partial \kappa}{\partial u} \in C^{2 r}(\Omega)$ and $f \in C^{d}([0,1])$, then using (1.5) we deduce that

$$
\tilde{z}_{n}^{M}-\varphi_{m}=\mathscr{K}_{m}^{\prime}\left(\varphi_{m}\right)\left(z_{n}^{M}-\varphi_{m}\right)+O\left(\max \left\{\tilde{h}^{d}, h^{3 r}\right\}^{2}\right)
$$

Our aim is to obtain an asymptotic series expansion for the first term on the right hand side of the above equation.

We first prove the following preliminary results which are needed later on.

Lemma 4.1. If $\frac{\partial \kappa}{\partial u} \in C^{3 r+3}(\Omega)$, then for $x \in B(\varphi, \delta)$ and for $1 \leq k \leq 4$,

$$
\begin{gathered}
\left\|\left(I-Q_{n}\right) \mathscr{K}_{m}^{(k)}(x)\right\|=O\left(h^{r}\right), \\
\left\|\mathscr{K}_{m}^{\prime}\left(\varphi_{m}\right)\left(I-Q_{n}\right) \mathscr{K}_{m}^{(k)}(x)\right\|=O\left(h^{2 r}\right)
\end{gathered}
$$

and

$$
\left\|\mathscr{K}_{m}^{\prime}\left(\varphi_{m}\right)\left(I-Q_{n}\right) \mathscr{K}_{m}^{\prime}\left(\varphi_{m}\right)\left(I-Q_{n}\right) \mathscr{K}_{m}^{\prime}(x)\right\|=O\left(h^{4 r}\right) .
$$

Proof. Note that for $1 \leq k \leq 4$ and for $v_{1}, \ldots, v_{k} \in C([0,1])$,

$$
\mathscr{K}_{m}^{(k)}(x)\left(v_{1}, \ldots, v_{k}\right)(s)=\tilde{h} \sum_{j=1}^{m} \sum_{i=1}^{\rho} \omega_{i} \frac{\partial^{k} \kappa}{\partial u^{k}}\left(s, \zeta_{i}^{j}, x\left(\zeta_{i}^{j}\right)\right) v_{1}\left(\zeta_{i}^{j}\right) \cdots v_{k}\left(\zeta_{i}^{j}\right)
$$

and for $p=1, \ldots, 2 r$,

$$
\left(\mathscr{K}_{m}^{(k)}(x)\left(v_{1}, \ldots, v_{k}\right)\right)^{(p)}(s)=\tilde{h} \sum_{j=1}^{m} \sum_{i=1}^{\rho} \omega_{i} \frac{\partial^{p+k} \kappa}{\partial s^{p} \partial u^{k}}\left(s, \zeta_{i}^{j}, x\left(\zeta_{i}^{j}\right)\right) v_{1}\left(\zeta_{i}^{j}\right) \cdots v_{k}\left(\zeta_{i}^{j}\right) .
$$

Hence, for $x \in B(\varphi, \delta)$,

$$
\left\|\left(\mathscr{K}_{m}^{(k)}(x)\left(v_{1}, \ldots, v_{k}\right)\right)^{(p)}\right\|_{\infty} \leq C_{5}\left(\sum_{i=1}^{\rho}\left|\omega_{i}\right|\right)\left\|v_{1}\right\|_{\infty} \ldots\left\|v_{k}\right\|_{\infty} .
$$

From the estimate (2.9),

$$
\begin{aligned}
\left\|\left(I-Q_{n}\right) \mathscr{K}_{m}^{(k)}(x)\left(v_{1}, \ldots, v_{k}\right)\right\|_{\infty} & \leq C_{4}\left\|\left(\mathscr{K}_{m}^{(k)}(x)\left(v_{1}, \ldots, v_{k}\right)\right)^{(r)}\right\|_{\infty} h^{r} \\
& \leq C_{4} C_{5}\left(\sum_{i=1}^{\rho}\left|\omega_{i}\right|\right)\left\|v_{1}\right\|_{\infty} \ldots\left\|v_{k}\right\|_{\infty} h^{r} .
\end{aligned}
$$


By taking the supremum over the set $\left\{\left(v_{1}, \ldots, v_{k}\right):\left\|v_{1}\right\|_{\infty} \leq 1, \ldots,\left\|v_{k}\right\|_{\infty} \leq 1\right\}$, we obtain (4.2).

We recall the following result from Kulkarni-Rakshit [7]: If $v \in C^{2 r}[0,1]$, then

$$
\left\|\mathscr{K}_{m}^{\prime}\left(\varphi_{m}\right)\left(I-Q_{n}\right) v\right\|_{\infty} \leq C_{6}\left\|\ell_{m}\right\|_{r, \infty}\|v\|_{2 r, \infty} h^{2 r}
$$

where

$$
\ell_{m}(s, t)=\frac{\partial \kappa}{\partial u}\left(s, t, \varphi_{m}(t)\right), s, t \in[0,1], \quad\left\|\ell_{m}\right\|_{r, \infty}=\max _{0 \leq i+j \leq r}\left\|D^{(i, j)} \ell_{m}\right\|_{\infty}
$$

and $C_{6}=\frac{1}{r !} 2^{r}\|\Psi\|_{\infty}\left(\sum_{i=1}^{\rho}\left|\omega_{i}\right|\right)$ is a constant independent of $n$. Since $\left\|\ell_{m}\right\|_{r, \infty} \leq C_{5}$, it follows that

$$
\left\|\mathscr{K}_{m}^{\prime}\left(\varphi_{m}\right)\left(I-Q_{n}\right) \mathscr{K}_{m}^{(k)}(x)\left(v_{1}, \ldots, v_{k}\right)\right\|_{\infty} \leq\left(C_{5}\right)^{2} C_{6}\left(\sum_{i=1}^{\rho}\left|\omega_{i}\right|\right)\left\|v_{1}\right\|_{\infty} \ldots\left\|v_{k}\right\|_{\infty} h^{2 r} .
$$

By taking the supremum over the set $\left\{\left(v_{1}, \ldots, v_{k}\right):\left\|v_{1}\right\|_{\infty} \leq 1, \ldots,\left\|v_{k}\right\|_{\infty} \leq 1\right\}$, we obtain (4.3).

In order to prove (4.4), we recall the following estimate from Kulkarni-Rakshit [7]: If $u \in C^{2 r}[0,1]$, then

$$
\left\|\mathscr{K}_{m}^{\prime}\left(\varphi_{m}\right)\left(I-Q_{n}\right) \mathscr{K}_{m}^{\prime}\left(\varphi_{m}\right)\left(I-Q_{n}\right) u\right\|_{\infty} \leq\left(C_{6}\right)^{2}\left\|\ell_{m}\right\|_{r, \infty}\left\|\ell_{m}\right\|_{3 r, \infty}\|u\|_{2 r, \infty} h^{4 r}
$$

Then for $v \in C[0,1]$,

$$
\begin{aligned}
\| \mathscr{K}_{m}^{\prime}\left(\varphi_{m}\right)(I & \left.-Q_{n}\right) \mathscr{K}_{m}^{\prime}\left(\varphi_{m}\right)\left(I-Q_{n}\right) \mathscr{K}_{m}^{\prime}(x) v \|_{\infty} \\
& \leq\left(C_{6}\right)^{2}\left\|\ell_{m}\right\|_{r, \infty}\left\|\ell_{m}\right\|_{3 r, \infty}\left\|\mathscr{K}_{m}^{\prime}(x) v\right\|_{2 r, \infty} h^{4 r} \\
& \leq\left(C_{5}\right)^{2}\left(C_{6}\right)^{2}\left(\sum_{i=1}^{\rho}\left|\omega_{i}\right|\right)\left\|\ell_{m}\right\|_{3 r, \infty}\|v\|_{\infty} h^{4 r} .
\end{aligned}
$$

The estimate (4.4) then follows by taking the supremum over the set $\left\{v \in C[0,1]:\|v\|_{\infty} \leq 1\right\}$.

From Proposition 4.2 of Kulkarni-Rakshit [7] we recall that for all $m$ big enough, $I-\mathscr{K}_{m}^{\prime}\left(\varphi_{m}\right)$ is invertible and that $\left\|\left(I-\mathscr{K}_{m}^{\prime}\left(\varphi_{m}\right)\right)^{-1}\right\| \leq C_{7}$, where $C_{7}$ is a constant independent of $m$. It can be easily checked that

$$
z_{n}^{M}-\varphi_{m}=-\left[I-\mathscr{K}_{m}^{\prime}\left(\varphi_{m}\right)\right]^{-1}\left\{\mathscr{K}_{m}\left(\varphi_{m}\right)-\mathscr{K}_{m}^{\prime}\left(\varphi_{m}\right) \varphi_{m}-\tilde{\mathscr{K}}_{n}^{M}\left(z_{n}^{M}\right)+\mathscr{K}_{m}^{\prime}\left(\varphi_{m}\right) z_{n}^{M}\right\}
$$


Let $L_{m}=\left(I-\mathscr{K}_{m}^{\prime}\left(\varphi_{m}\right)\right)^{-1}$. We write

$$
\begin{aligned}
\mathscr{K}_{m}^{\prime}\left(\varphi_{m}\right) & \left(z_{n}^{M}-\varphi_{m}\right) \\
= & -L_{m} \mathscr{K}_{m}^{\prime}\left(\varphi_{m}\right)\left\{\mathscr{K}_{m}\left(\varphi_{m}\right)-\tilde{\mathscr{K}}_{n}^{M}\left(\varphi_{m}\right)\right\} \\
& +L_{m} \mathscr{K}_{m}^{\prime}\left(\varphi_{m}\right)\left\{\tilde{\mathscr{K}}_{n}^{M}\left(z_{n}^{M}\right)-\tilde{\mathscr{K}}_{n}^{M}\left(\varphi_{m}\right)-\left(\tilde{\mathscr{K}}_{n}^{M}\right)^{\prime}\left(\varphi_{m}\right)\left(z_{n}^{M}-\varphi_{m}\right)\right\} \\
& +L_{m} \mathscr{K}_{m}^{\prime}\left(\varphi_{m}\right)\left\{\left(\left(\tilde{\mathscr{K}}_{n}^{M}\right)^{\prime}\left(\varphi_{m}\right)-\mathscr{K}_{m}^{\prime}\left(\varphi_{m}\right)\right)\left(z_{n}^{M}-\varphi_{m}\right)\right\} .
\end{aligned}
$$

We obtain an asymptotic expansion for the first term on the right hand side of (4.6) and show that the second and the third terms are of the order of $h^{2 r} \max \left\{\tilde{h}^{d}, h^{2 r+2}\right\}$. The following two lemmas are needed to obtain the results for the first term.

From now onwards we assume that

$$
d \geq 2 r, \frac{\partial \kappa}{\partial u} \in C^{\max \{d, 3 r+3\}}(\Omega) \text { and } f \in C^{\max \{d, 2 r+2\}}[0,1]
$$

Lemma 4.2. If $d \geq 2 r, \frac{\partial \kappa}{\partial u} \in C^{\max \{d, 3 r+3\}}(\Omega)$ and $f \in C^{\max \{d, 2 r+2\}}[0,1]$, then

$$
L_{m} \mathscr{K}_{m}^{\prime}\left(\varphi_{m}\right)\left(I-Q_{n}\right) \mathscr{K}_{m}^{\prime}\left(\varphi_{m}\right)\left(Q_{n}-I\right) \varphi_{m}=U(T(\varphi)) h^{4 r}+O\left(h^{2 r} \max \left\{\tilde{h}^{d}, h^{2 r+2}\right\}\right)
$$

and

$$
L_{m} \mathscr{K}_{m}^{\prime}\left(\varphi_{m}\right)\left(I-Q_{n}\right) \mathscr{K}_{m}^{\prime \prime}\left(\varphi_{m}\right)\left(\left(Q_{n}-I\right) \varphi_{m}\right)^{2}=U\left(V_{1}(\varphi)\right) h^{4 r}+O\left(h^{2 r} \max \left\{\tilde{h}^{d}, h^{2 r+2}\right\}\right) .
$$

Proof. Using the resolvent identity, we write

$$
\begin{aligned}
L_{m}= & {\left[I-\mathscr{K}^{\prime}(\varphi)\right]^{-1}+\left[I-\mathscr{K}^{\prime}(\varphi)\right]^{-1}\left[\mathscr{K}_{m}^{\prime}\left(\varphi_{m}\right)-\mathscr{K}_{m}^{\prime}(\varphi)\right] L_{m} } \\
& +\left[I-\mathscr{K}^{\prime}(\varphi)\right]^{-1}\left[\mathscr{K}_{m}^{\prime}(\varphi)-\mathscr{K}^{\prime}(\varphi)\right] L_{m} .
\end{aligned}
$$

Let

$$
\begin{aligned}
y_{n}= & \mathscr{K}_{m}^{\prime}\left(\varphi_{m}\right)\left(I-Q_{n}\right) \mathscr{K}_{m}^{\prime}\left(\varphi_{m}\right)\left(Q_{n}-I\right) \varphi_{m} \\
= & \mathscr{K}_{m}^{\prime}\left(\varphi_{m}\right)\left(I-Q_{n}\right) \mathscr{K}_{m}^{\prime}\left(\varphi_{m}\right)\left(Q_{n}-I\right) \varphi \\
& +\mathscr{K}_{m}^{\prime}\left(\varphi_{m}\right)\left(I-Q_{n}\right) \mathscr{K}_{m}^{\prime}\left(\varphi_{m}\right)\left(Q_{n}-I\right)\left(\varphi_{m}-\varphi\right) .
\end{aligned}
$$


From (2.6), (4.3) and (4.5), it follows that

$$
\left\|y_{n}\right\|_{\infty}=O\left(h^{2 r} \max \left\{\tilde{h}^{d}, h^{2 r}\right\}\right)=O\left(h^{4 r}\right)
$$

Note that

$$
\begin{aligned}
L_{m} y_{n}= & {\left[I-\mathscr{K}^{\prime}(\varphi)\right]^{-1} y_{n} } \\
& +\left[I-\mathscr{K}^{\prime}(\varphi)\right]^{-1}\left[\mathscr{K}_{m}^{\prime}\left(\varphi_{m}\right)-\mathscr{K}_{m}{ }^{\prime}(\varphi)\right] L_{m} y_{n} \\
& +\left[I-\mathscr{K}^{\prime}(\varphi)\right]^{-1}\left[\mathscr{K}_{m}^{\prime}(\varphi)-\mathscr{K}^{\prime}(\varphi)\right] L_{m} y_{n} .
\end{aligned}
$$

Consider the first term

$$
\begin{aligned}
{\left[I-\mathscr{K}^{\prime}(\varphi)\right]^{-1} y_{n} } & =\left[I-\mathscr{K}^{\prime}(\varphi)\right]^{-1} \mathscr{K}^{\prime}(\varphi)\left(I-Q_{n}\right) \mathscr{K}_{m}^{\prime}\left(\varphi_{m}\right)\left(Q_{n} \varphi_{m}-\varphi_{m}\right) \\
& +\left[I-\mathscr{K}^{\prime}(\varphi)\right]^{-1}\left(\mathscr{K}_{m}^{\prime}\left(\varphi_{m}\right)-\mathscr{K}^{\prime}(\varphi)\right)\left(I-Q_{n}\right) \mathscr{K}_{m}^{\prime}\left(\varphi_{m}\right)\left(Q_{n} \varphi_{m}-\varphi_{m}\right)
\end{aligned}
$$

Note that from (3.2) and (3.4),

$$
\begin{aligned}
{\left[I-\mathscr{K}^{\prime}(\varphi)\right]^{-1} \mathscr{K}^{\prime}(\varphi)\left(I-Q_{n}\right) \mathscr{K}_{m}^{\prime}\left(\varphi_{m}\right)\left(Q_{n} \varphi_{m}-\varphi_{m}\right) } \\
=U(T(\varphi)) h^{4 r}+O\left(h^{2 r} \max \left\{\tilde{h}^{d}, h^{2 r+2}\right\}\right) .
\end{aligned}
$$

On the other hand,

$$
\begin{aligned}
\left(I-Q_{n}\right) \mathscr{K}_{m}^{\prime}\left(\varphi_{m}\right)\left(Q_{n} \varphi_{m}-\varphi_{m}\right) & =\left(I-Q_{n}\right) \mathscr{K}_{m}^{\prime}(\varphi)\left(Q_{n} \varphi-\varphi\right) \\
& +\left(I-Q_{n}\right) \mathscr{K}_{m}^{\prime}(\varphi)\left(Q_{n}-I\right)\left(\varphi_{m}-\varphi\right) \\
& +\left(I-Q_{n}\right)\left(\mathscr{K}_{m}^{\prime}\left(\varphi_{m}\right)-\mathscr{K}_{m}^{\prime}(\varphi)\right)\left(Q_{n} \varphi_{m}-\varphi_{m}\right) .
\end{aligned}
$$

From Kulkarni-Rakshit [7, Proposition 2.3], we have

$$
\left\|\left(I-Q_{n}\right) \mathscr{K}_{m}^{\prime}(\varphi)\left(Q_{n} \varphi-\varphi\right)\right\|_{\infty}=O\left(h^{3 r}\right)
$$

Combining the above estimate with the estimates (2.6), (2.10), (3.3) and (4.2), we obtain

$$
\left\|\left[I-\mathscr{K}^{\prime}(\varphi)\right]^{-1}\left(\mathscr{K}_{m}^{\prime}\left(\varphi_{m}\right)-\mathscr{K}^{\prime}(\varphi)\right)\left(I-Q_{n}\right) \mathscr{K}_{m}^{\prime}\left(\varphi_{m}\right)\left(Q_{n} \varphi_{m}-\varphi_{m}\right)\right\|_{\infty}=O\left(\tilde{h}^{d} h^{3 r}\right) .
$$

Thus,

$$
\left[I-\mathscr{K}^{\prime}(\varphi)\right]^{-1} y_{n}=U(T(\varphi)) h^{4 r}+O\left(h^{2 r} \max \left\{\tilde{h}^{d}, h^{2 r+2}\right\}\right) .
$$


Using the estimate (4.9), it then follows that the second term of (4.10) is of the order of $\tilde{h}^{d} h^{4 r}$. We write the third term of (4.10) as

$$
\left[I-\mathscr{K}^{\prime}(\varphi)\right]^{-1}\left[\mathscr{K}_{m}^{\prime}(\varphi)-\mathscr{K}^{\prime}(\varphi)\right] \mathscr{K}_{m}^{\prime}\left(\varphi_{m}\right) L_{m}\left(I-Q_{n}\right) \mathscr{K}_{m}^{\prime}\left(\varphi_{m}\right)\left(Q_{n}-I\right)\left(\varphi_{m}-\varphi+\varphi\right) .
$$

From (2.7), for $v \in C[0,1]$,

$$
\left\|\left(\mathscr{K}_{m}^{\prime}(\varphi)-\mathscr{K}^{\prime}(\varphi)\right) \mathscr{K}_{m}^{\prime}\left(\varphi_{m}\right) v\right\|_{\infty} \leq C_{2}\left\|\mathscr{K}_{m}^{\prime}\left(\varphi_{m}\right) v\right\|_{d, \infty} \tilde{h}^{d} \leq C_{2} C_{5}\left(\sum_{i=1}^{\rho}\left|\omega_{i}\right|\right)\|v\|_{\infty} \tilde{h}^{d} .
$$

It follows that

$$
\left\|\left(\mathscr{K}_{m}^{\prime}(\varphi)-\mathscr{K}^{\prime}(\varphi)\right) \mathscr{K}_{m}^{\prime}\left(\varphi_{m}\right)\right\|=O\left(\tilde{h}^{d}\right)
$$

whereas as in Lemma 4.1, it can be proved that

$$
\left\|\left(I-Q_{n}\right) \mathscr{K}_{m}^{\prime}\left(\varphi_{m}\right)\left(I-Q_{n}\right) \varphi\right\|_{\infty}=O\left(h^{3 r}\right)
$$

Thus the third term of (4.10) is of the order of $\tilde{h}^{d} h^{3 r}$.

Hence

$$
\begin{aligned}
L_{m} y_{n} & =L_{m} \mathscr{K}_{m}^{\prime}\left(\varphi_{m}\right)\left(I-Q_{n}\right) \mathscr{K}_{m}^{\prime}\left(\varphi_{m}\right)\left(Q_{n} \varphi_{m}-\varphi_{m}\right) \\
& =U(T(\varphi)) h^{4 r}+O\left(h^{2 r} \max \left\{\tilde{h}^{d}, h^{2 r+2}\right\}\right),
\end{aligned}
$$

which proves (4.7). The proof of (4.8) is similar.

Lemma 4.3. If $d \geq 2 r, \frac{\partial \kappa}{\partial u} \in C^{\max \{d, 3 r+3\}}(\Omega)$ and $f \in C^{\max \{d, 2 r+2\}}[0,1]$, then

$$
\left\|L_{m} \mathscr{K}_{m}^{\prime}\left(\varphi_{m}\right)\left(I-Q_{n}\right) \mathscr{K}_{m}^{(3)}\left(\varphi_{m}\right)\left(Q_{n} \varphi_{m}-\varphi_{m}\right)^{3}\right\|_{\infty}=O\left(h^{4 r+2}\right) .
$$

Proof. Let

$$
w_{n}=\mathscr{K}_{m}^{\prime}\left(\varphi_{m}\right)\left(I-Q_{n}\right) \mathscr{K}_{m}^{(3)}\left(\varphi_{m}\right)\left(Q_{n} \varphi_{m}-\varphi_{m}\right)^{3} .
$$

Then using (2.10) and (4.3), we obtain

$$
\left\|w_{n}\right\|_{\infty}=O\left(h^{5 r}\right) .
$$

As in Lemma 4.2, we write

$$
\begin{aligned}
L_{m} w_{n} & =\left[I-\mathscr{K}^{\prime}(\varphi)\right]^{-1} w_{n} \\
& +\left[I-\mathscr{K}^{\prime}(\varphi)\right]^{-1}\left[\mathscr{K}_{m}^{\prime}\left(\varphi_{m}\right)-\mathscr{K}_{m}^{\prime}(\varphi)\right] L_{m} w_{n} \\
& +\left[I-\mathscr{K}^{\prime}(\varphi)\right]^{-1}\left[\mathscr{K}_{m}^{\prime}(\varphi)-\mathscr{K}^{\prime}(\varphi)\right] L_{m} w_{n}
\end{aligned}
$$


Note that

$$
\begin{aligned}
{\left[I-\mathscr{K}^{\prime}(\varphi)\right]^{-1} w_{n} } & =\left[I-\mathscr{K}^{\prime}(\varphi)\right]^{-1} \mathscr{K}^{\prime}(\varphi)\left(I-Q_{n}\right) \mathscr{K}_{m}^{(3)}\left(\varphi_{m}\right)\left(Q_{n} \varphi_{m}-\varphi_{m}\right)^{3} \\
& +\left[I-\mathscr{K}^{\prime}(\varphi)\right]^{-1}\left(\mathscr{K}_{m}^{\prime}\left(\varphi_{m}\right)-\mathscr{K}^{\prime}(\varphi)\right)\left(I-Q_{n}\right) \mathscr{K}_{m}^{(3)}\left(\varphi_{m}\right)\left(Q_{n} \varphi_{m}-\varphi_{m}\right)^{3} .
\end{aligned}
$$

Using the asymptotic expansions (3.2) and (3.6) we obtain

$$
\begin{array}{r}
{\left[I-\mathscr{K}^{\prime}(\varphi)\right]^{-1} \mathscr{K}^{\prime}(\varphi)\left(I-Q_{n}\right) \mathscr{K}_{m}^{(3)}\left(\varphi_{m}\right)\left(Q_{n} \varphi_{m}-\varphi_{m}\right)^{3}} \\
=U\left(V_{2}(\varphi)\right) h^{5 r}+O\left(h^{2 r} \max \left\{\tilde{h}^{d}, h^{3 r+1}\right\}\right) .
\end{array}
$$

If $r=1$, then $V_{2}(\varphi)=0$ and if $r \geq 2$, then $5 r \geq 4 r+2$. Hence it follows that

$$
\left\|\left[I-\mathscr{K}^{\prime}(\varphi)\right]^{-1} \mathscr{K}^{\prime}(\varphi)\left(I-Q_{n}\right) \mathscr{K}_{m}^{(3)}\left(\varphi_{m}\right)\left(Q_{n} \varphi_{m}-\varphi_{m}\right)^{3}\right\|_{\infty}=O\left(h^{4 r+2}\right)
$$

On the other hand, using (2.7), (2.10), (3.3) and (4.2) we obtain

$$
\left\|\left[I-\mathscr{K}^{\prime}(\varphi)\right]^{-1}\left(\mathscr{K}_{m}^{\prime}\left(\varphi_{m}\right)-\mathscr{K}^{\prime}(\varphi)\right)\left(I-Q_{n}\right) \mathscr{K}_{m}^{(3)}\left(\varphi_{m}\right)\left(Q_{n} \varphi_{m}-\varphi_{m}\right)^{3}\right\|_{\infty}=O\left(\tilde{h}^{d} h^{3 r}\right)
$$

Sine $d \geq 2 r$, it follows that

$$
\left\|\left[I-\mathscr{K}^{\prime}(\varphi)\right]^{-1} w_{n}\right\|_{\infty}=O\left(h^{4 r+2}\right)
$$

Using the estimates (3.3) and (4.12), we see that

$$
\left\|\left[I-\mathscr{K}^{\prime}(\varphi)\right]^{-1}\left[\mathscr{K}_{m}^{\prime}\left(\varphi_{m}\right)-\mathscr{K}_{m}^{\prime}(\varphi)\right] L_{m} w_{n}\right\|_{\infty}=O\left(\tilde{h}^{d} h^{5 r}\right)
$$

We write the third term of (4.13) as

$$
\left[I-\mathscr{K}^{\prime}(\varphi)\right]^{-1}\left[\mathscr{K}_{m}^{\prime}(\varphi)-\mathscr{K}^{\prime}(\varphi)\right] \mathscr{K}_{m}^{\prime}\left(\varphi_{m}\right) L_{m}\left(I-Q_{n}\right) \mathscr{K}_{m}^{(3)}\left(\varphi_{m}\right)\left(Q_{n} \varphi_{m}-\varphi_{m}\right)^{3}
$$

From (2.10), (4.2) and (4.11), it follows that the above term is of the order of $\tilde{h}^{d} h^{4 r}$. Thus,

$$
\left\|\left[I-\mathscr{K}^{\prime}(\varphi)\right]^{-1}\left[\mathscr{K}_{m}^{\prime}(\varphi)-\mathscr{K}^{\prime}(\varphi)\right] L_{m} w_{n}\right\|_{\infty}=O\left(\tilde{h}^{d} h^{4 r}\right)
$$

Since $d \geq 2 r$, it follows from (4.13) - (4.17) that

$$
\left\|L_{m} w_{n}\right\|_{\infty}=\left\|L_{m} \mathscr{K}_{m}^{\prime}\left(\varphi_{m}\right)\left(I-Q_{n}\right) \mathscr{K}_{m}^{(3)}\left(\varphi_{m}\right)\left(Q_{n} \varphi_{m}-\varphi_{m}\right)^{3}\right\|_{\infty}=O\left(h^{4 r+2}\right)
$$

This completes the proof. 
We now obtain the asymptotic expansion for the first term of (4.6).

Proposition 4.4. If $d \geq 2 r, \frac{\partial \kappa}{\partial u} \in C^{\max \{d, 3 r+3\}}(\Omega)$ and $f \in C^{\max \{d, 2 r+2\}}[0,1]$, then

$$
\begin{aligned}
L_{m} \mathscr{K}_{m}^{\prime}\left(\varphi_{m}\right) & \left\{\mathscr{K}_{m}\left(\varphi_{m}\right)-\tilde{\mathscr{K}}_{n}^{M}\left(\varphi_{m}\right)\right\} \\
& =-U\left(T(\varphi)+\frac{V_{1}(\varphi)}{2}\right) h^{4 r}+O\left(h^{2 r} \max \left\{\tilde{h}^{d}, h^{2 r+2}\right\}\right) .
\end{aligned}
$$

Proof. Note that

$$
\mathscr{K}_{m}\left(\varphi_{m}\right)-\tilde{\mathscr{K}}_{n}^{M}\left(\varphi_{m}\right)=\left(I-Q_{n}\right)\left(\mathscr{K}_{m}\left(\varphi_{m}\right)-\mathscr{K}_{m}\left(Q_{n} \varphi_{m}\right)\right)
$$

By Taylor's theorem,

$$
\begin{aligned}
\mathscr{K}_{m}\left(Q_{n} \varphi_{m}\right)-\mathscr{K}_{m}\left(\varphi_{m}\right) & =\mathscr{K}_{m}^{\prime}\left(\varphi_{m}\right)\left(Q_{n} \varphi_{m}-\varphi_{m}\right)+\frac{\mathscr{K}_{m}^{\prime \prime}\left(\varphi_{m}\right)}{2}\left(Q_{n} \varphi_{m}-\varphi_{m}\right)^{2} \\
& +\frac{\mathscr{K}_{m}^{(3)}\left(\varphi_{m}\right)}{6}\left(Q_{n} \varphi_{m}-\varphi_{m}\right)^{3}+\frac{\mathscr{K}_{m}^{(4)}\left(\xi_{m}\right)}{24}\left(Q_{n} \varphi_{m}-\varphi_{m}\right)^{4}
\end{aligned}
$$

where $\xi_{m} \in B(\varphi, \delta)$. Hence

$$
\begin{aligned}
L_{m} \mathscr{K}_{m}^{\prime}\left(\varphi_{m}\right)\left\{\mathscr{K}_{m}\left(\varphi_{m}\right)-\tilde{\mathscr{K}}_{n}^{M}\left(\varphi_{m}\right)\right\}= & -L_{m} \mathscr{K}_{m}^{\prime}\left(\varphi_{m}\right)\left(I-Q_{n}\right) \mathscr{K}_{m}^{\prime}\left(\varphi_{m}\right)\left(Q_{n} \varphi_{m}-\varphi_{m}\right) \\
& -\frac{1}{2} L_{m} \mathscr{K}_{m}^{\prime}\left(\varphi_{m}\right)\left(I-Q_{n}\right) \mathscr{K}_{m}^{\prime \prime}\left(\varphi_{m}\right)\left(Q_{n} \varphi_{m}-\varphi_{m}\right)^{2} \\
& -\frac{1}{6} L_{m} \mathscr{K}_{m}^{\prime}\left(\varphi_{m}\right)\left(I-Q_{n}\right) \mathscr{K}_{m}^{(3)}\left(\varphi_{m}\right)\left(Q_{n} \varphi_{m}-\varphi_{m}\right)^{3} \\
& -\frac{1}{24} L_{m} \mathscr{K}_{m}^{\prime}\left(\varphi_{m}\right)\left(I-Q_{n}\right) \mathscr{K}_{m}^{(4)}\left(\xi_{m}\right)\left(Q_{n} \varphi_{m}-\varphi_{m}\right)^{4} .
\end{aligned}
$$

Using (2.10) and (4.3) we deduce that

$$
\left\|L_{m} \mathscr{K}_{m}^{\prime}\left(\varphi_{m}\right)\left(I-Q_{n}\right) \mathscr{K}_{m}^{(4)}\left(\xi_{m}\right)\left(Q_{n} \varphi_{m}-\varphi_{m}\right)^{4}\right\|_{\infty}=O\left(h^{6 r}\right)
$$

Using the above estimate, Lemma 4.2 and Lemma 4.3, we obtain

$$
L_{m} \mathscr{K}_{m}^{\prime}\left(\varphi_{m}\right)\left\{\mathscr{K}_{m}\left(\varphi_{m}\right)-\tilde{\mathscr{K}}_{n}^{M}\left(\varphi_{m}\right)\right\}=-U\left(T(\varphi)+\frac{V_{1}(\varphi)}{2}\right) h^{4 r}+O\left(h^{4 r+2}\right)
$$

which completes the proof.

We quote the following result from Kulkarni-Rakshit [7, Proposition 4.6]:

$$
\left\|\tilde{\mathscr{K}}_{n}^{M}\left(z_{n}^{M}\right)-\tilde{\mathscr{K}}_{n}^{M}\left(\varphi_{m}\right)-\left(\tilde{\mathscr{K}}_{n}^{M}\right)^{\prime}\left(\varphi_{m}\right)\left(z_{n}^{M}-\varphi_{m}\right)\right\|_{\infty}=O\left(\max \left\{\tilde{h}^{d}, h^{3 r}\right\}^{2}\right) .
$$


It follows that the second term on the right hand side of (4.6) is of the order of $\max \left\{\tilde{h}^{d}, h^{3 r}\right\}^{2}$. We now want obtain the order of convergence of the third term on the right hand side of (4.6). For this purpose we first prove the following result.

Lemma 4.5. If $d \geq 2 r, \frac{\partial \kappa}{\partial u} \in C^{\max \{d, 3 r+3\}}(\Omega)$ and $f \in C^{\max \{d, 2 r+2\}}[0,1]$, then

$$
\begin{gathered}
\left\|\mathscr{K}_{m}^{\prime}\left(\varphi_{m}\right)\left(I-Q_{n}\right) \mathscr{K}_{m}^{\prime}\left(\varphi_{m}\right)\left(I-Q_{n}\right)\left[\tilde{\mathscr{K}}_{n}^{M}\left(\varphi_{m}\right)-\mathscr{K}_{m}\left(\varphi_{m}\right)\right]\right\|_{\infty}=O\left(h^{6 r}\right), \\
\left\|\mathscr{K}_{m}^{\prime}\left(\varphi_{m}\right)\left(I-Q_{n}\right) \mathscr{K}_{m}^{\prime}\left(\varphi_{m}\right)\left(I-Q_{n}\right)\left(\tilde{\mathscr{K}}_{n}^{M}\right)^{\prime}\left(\varphi_{m}\right)\right\|=O\left(h^{3 r}\right)
\end{gathered}
$$

Proof. Note that

$$
\begin{aligned}
\tilde{\mathscr{K}}_{n}^{M}\left(\varphi_{m}\right)-\mathscr{K}_{m}\left(\varphi_{m}\right) & =\left(I-Q_{n}\right)\left(\mathscr{K}_{m}\left(Q_{n} \varphi_{m}\right)-\mathscr{K}_{m}\left(\varphi_{m}\right)\right) \\
& =\left(I-Q_{n}\right) \mathscr{K}_{m}^{\prime}\left(\xi_{m}\right)\left(Q_{n} \varphi_{m}-\varphi_{m}\right),
\end{aligned}
$$

where $\xi_{m} \in B(\varphi, \delta)$. Hence

$$
\begin{aligned}
\| \mathscr{K}_{m}^{\prime}\left(\varphi_{m}\right)(I & \left.-Q_{n}\right) \mathscr{K}_{m}^{\prime}\left(\varphi_{m}\right)\left(I-Q_{n}\right) \mathscr{K}_{m}^{\prime}\left(\xi_{m}\right)\left(Q_{n} \varphi_{m}-\varphi_{m}\right) \|_{\infty} \\
& \leq\left\|\mathscr{K}_{m}^{\prime}\left(\varphi_{m}\right)\left(I-Q_{n}\right) \mathscr{K}_{m}^{\prime}\left(\varphi_{m}\right)\left(I-Q_{n}\right) \mathscr{K}_{m}^{\prime}\left(\xi_{m}\right)\left(Q_{n} \varphi-\varphi\right)\right\|_{\infty} \\
& +\left\|\mathscr{K}_{m}^{\prime}\left(\varphi_{m}\right)\left(I-Q_{n}\right) \mathscr{K}_{m}^{\prime}\left(\varphi_{m}\right)\left(I-Q_{n}\right) \mathscr{K}_{m}^{\prime}\left(\xi_{m}\right)\left(Q_{n}-I\right)\left(\varphi_{m}-\varphi\right)\right\|_{\infty}
\end{aligned}
$$

Recall from (4.5) that

$$
\begin{aligned}
\| \mathscr{K}_{m}^{\prime}\left(\varphi_{m}\right)(I & \left.-Q_{n}\right) \mathscr{K}_{m}^{\prime}\left(\varphi_{m}\right)\left(I-Q_{n}\right) \mathscr{K}_{m}^{\prime}\left(\xi_{m}\right)\left(Q_{n} \varphi-\varphi\right) \|_{\infty} \\
& \leq\left(C_{6}\right)^{2}\left\|\ell_{m}\right\|_{r, \infty}\left\|\ell_{m}\right\|_{3 r, \infty}\left\|\mathscr{K}_{m}^{\prime}\left(\xi_{m}\right)\left(Q_{n} \varphi-\varphi\right)\right\|_{2 r, \infty} h^{4 r}
\end{aligned}
$$

and it can be checked that

$$
\left\|\mathscr{K}_{m}^{\prime}\left(\xi_{m}\right)\left(Q_{n} \varphi-\varphi\right)\right\|_{2 r, \infty} \leq C_{5} C_{6}\|\varphi\|_{2 r, \infty} h^{2 r}
$$

Thus,

$$
\left\|\mathscr{K}_{m}^{\prime}\left(\varphi_{m}\right)\left(I-Q_{n}\right) \mathscr{K}_{m}^{\prime}\left(\varphi_{m}\right)\left(I-Q_{n}\right) \mathscr{K}_{m}^{\prime}\left(\xi_{m}\right)\left(Q_{n} \varphi-\varphi\right)\right\|_{\infty}=O\left(h^{6 r}\right)
$$


On the other hand, since $d \geq 2 r$ and $\tilde{h} \leq h$, from (2.6) and (4.4),

$$
\begin{aligned}
\left\|\mathscr{K}_{m}^{\prime}\left(\varphi_{m}\right)\left(I-Q_{n}\right) \mathscr{K}_{m}^{\prime}\left(\varphi_{m}\right)\left(I-Q_{n}\right) \mathscr{K}_{m}^{\prime}\left(\xi_{m}\right)\left(Q_{n}-I\right)\left(\varphi_{m}-\varphi\right)\right\|_{\infty} \\
\quad \leq\left\|\mathscr{K}_{m}^{\prime}\left(\varphi_{m}\right)\left(I-Q_{n}\right) \mathscr{K}_{m}^{\prime}\left(\varphi_{m}\right)\left(I-Q_{n}\right) \mathscr{K}_{m}^{\prime}\left(\xi_{m}\right)\right\|\left(1+\left\|Q_{n}\right\|\right)\left\|\varphi_{m}-\varphi\right\|_{\infty} \\
\quad=O\left(h^{4 r} \tilde{h}^{d}\right)=O\left(h^{6 r}\right) .
\end{aligned}
$$

The estimate (4.18) then follows from (4.20), (4.21) and (4.22).

In order to prove (4.19), recall that

$$
\left(\tilde{\mathscr{K}}_{n}^{M}\right)^{\prime}\left(\varphi_{m}\right)=Q_{n} \mathscr{K}_{m}^{\prime}\left(\varphi_{m}\right)+\left(I-Q_{n}\right) \mathscr{K}_{m}^{\prime}\left(Q_{n} \varphi_{m}\right) Q_{n}
$$

Hence

$$
\begin{aligned}
\mathscr{K}_{m}^{\prime}\left(\varphi_{m}\right)(I- & \left.Q_{n}\right) \mathscr{K}_{m}^{\prime}\left(\varphi_{m}\right)\left(I-Q_{n}\right)\left(\tilde{\mathscr{K}}_{n}^{M}\right)^{\prime}\left(\varphi_{m}\right) \\
& =\mathscr{K}_{m}^{\prime}\left(\varphi_{m}\right)\left(I-Q_{n}\right) \mathscr{K}_{m}^{\prime}\left(\varphi_{m}\right)\left(I-Q_{n}\right)\left(\mathscr{K}_{m}^{\prime}\left(Q_{n} \varphi_{m}\right)-\mathscr{K}_{m}^{\prime}\left(\varphi_{m}\right)\right) Q_{n} \\
& +\mathscr{K}_{m}^{\prime}\left(\varphi_{m}\right)\left(I-Q_{n}\right) \mathscr{K}_{m}^{\prime}\left(\varphi_{m}\right)\left(I-Q_{n}\right) \mathscr{K}_{m}^{\prime}\left(\varphi_{m}\right) Q_{n}
\end{aligned}
$$

The result (4.19) follows from (2.10), (3.3), (4.3) and (4.4).

We now obtain the order of convergence of the third term in (4.6).

Proposition 4.6. If $d \geq 2 r, \frac{\partial \kappa}{\partial u} \in C^{\max \{d, 3 r+3\}}(\Omega)$ and $f \in C^{\max \{d, 2 r+2\}}[0,1]$, then

$$
\left\|\mathscr{K}_{m}^{\prime}\left(\varphi_{m}\right)\left\{\left(\left(\tilde{\mathscr{K}}_{n}^{M}\right)^{\prime}\left(\varphi_{m}\right)-\mathscr{K}_{m}^{\prime}\left(\varphi_{m}\right)\right)\left(z_{n}^{M}-\varphi_{m}\right)\right\}\right\|_{\infty}=O\left(h^{3 r} \max \left\{\tilde{h}^{d}, h^{3 r}\right\}\right) .
$$

Proof. Note that

$$
\begin{aligned}
\left(\tilde{\mathscr{K}}_{n}^{M}\right)^{\prime}\left(\varphi_{m}\right)-\mathscr{K}_{m}^{\prime}\left(\varphi_{m}\right)= & \left(I-Q_{n}\right)\left(\mathscr{K}_{m}^{\prime}\left(Q_{n} \varphi_{m}\right)-\mathscr{K}_{m}^{\prime}\left(\varphi_{m}\right)\right) Q_{n} \\
& -\left(I-Q_{n}\right) \mathscr{K}_{m}^{\prime}\left(\varphi_{m}\right)\left(I-Q_{n}\right) .
\end{aligned}
$$

By Taylor's theorem,

$$
\left(\mathscr{K}_{m}^{\prime}\left(Q_{n} \varphi_{m}\right)-\mathscr{K}_{m}^{\prime}\left(\varphi_{m}\right)\right) Q_{n}\left(z_{n}^{M}-\varphi_{m}\right)=\mathscr{K}_{m}^{\prime \prime}\left(\xi_{m}\right)\left(Q_{n} \varphi_{m}-\varphi_{m}, Q_{n}\left(z_{n}^{M}-\varphi_{m}\right)\right)
$$

where $\xi_{m} \in B(\varphi, \delta)$. Hence from (1.5), (2.10) and (4.3), we obtain

$$
\begin{aligned}
\| \mathscr{K}_{m}^{\prime}\left(\varphi_{m}\right)(I & \left.-Q_{n}\right) \mathscr{K}_{m}^{\prime \prime}\left(\xi_{m}\right)\left(Q_{n} \varphi_{m}-\varphi_{m}, Q_{n}\left(z_{n}^{M}-\varphi_{m}\right)\right) \|_{\infty} \\
& \leq\left\|\mathscr{K}_{m}^{\prime}\left(\varphi_{m}\right)\left(I-Q_{n}\right) \mathscr{K}_{m}^{\prime \prime}\left(\xi_{m}\right)\right\|\left\|Q_{n} \varphi_{m}-\varphi_{m}\right\|_{\infty}\left\|Q_{n}\right\|\left\|z_{n}^{M}-\varphi_{m}\right\|_{\infty} \\
& =O\left(h^{3 r} \max \left\{\tilde{h}^{d}, h^{3 r}\right\}\right) .
\end{aligned}
$$


Note that

$$
\begin{aligned}
& \mathscr{K}_{m}^{\prime}\left(\varphi_{m}\right)\left(I-Q_{n}\right) \mathscr{K}_{m}^{\prime}\left(\varphi_{m}\right)\left(I-Q_{n}\right)\left(z_{n}^{M}-\varphi_{m}\right) \\
& =\mathscr{K}_{m}^{\prime}\left(\varphi_{m}\right)\left(I-Q_{n}\right) \mathscr{K}_{m}^{\prime}\left(\varphi_{m}\right)\left(I-Q_{n}\right) \\
& \quad \times \quad\left[\tilde{\mathscr{K}}_{n}^{M}\left(z_{n}^{M}\right)-\tilde{\mathscr{K}}_{n}^{M}\left(\varphi_{m}\right)-\left(\tilde{\mathscr{K}}_{n}^{M}\right)^{\prime}\left(\varphi_{m}\right)\left(z_{n}^{M}-\varphi_{m}\right)\right] \\
& \quad+\mathscr{K}_{m}^{\prime}\left(\varphi_{m}\right)\left(I-Q_{n}\right) \mathscr{K}_{m}^{\prime}\left(\varphi_{m}\right)\left(I-Q_{n}\right)\left[\tilde{\mathscr{K}}_{n}^{M}\left(\varphi_{m}\right)-\mathscr{K}_{m}\left(\varphi_{m}\right)\right] \\
& +\mathscr{K}_{m}^{\prime}\left(\varphi_{m}\right)\left(I-Q_{n}\right) \mathscr{K}_{m}^{\prime}\left(\varphi_{m}\right)\left(I-Q_{n}\right)\left[\left(\tilde{\mathscr{K}}_{n}^{M}\right)^{\prime}\left(\varphi_{m}\right)\left(z_{n}^{M}-\varphi_{m}\right)\right] .
\end{aligned}
$$

By (4.3) and (4.17), the first term on the right hand side of the above equation is of the order of $h^{2 r} \max \left\{\tilde{h}^{d}, h^{3 r}\right\}^{2}$. By (4.18) of Lemma 4.5, the second term is of the order of $h^{6 r}$. By (4.19) of Lemma 4.5 and by (1.5), the third term is of the order of $h^{3 r} \max \left\{\tilde{h}^{d}, h^{3 r}\right\}$. It follows that

$$
\left\|\mathscr{K}_{m}^{\prime}\left(\varphi_{m}\right)\left(I-Q_{n}\right) \mathscr{K}_{m}^{\prime}\left(\varphi_{m}\right)\left(I-Q_{n}\right)\left(z_{n}^{M}-\varphi_{m}\right)\right\|_{\infty}=O\left(h^{3 r} \max \left\{\tilde{h}^{d}, h^{3 r}\right\}\right) .
$$

The required result follows from (4.23) and the above estimate.

We now prove our main result.

Theorem 4.7. If $d \geq 2 r, \frac{\partial \kappa}{\partial u} \in C^{\max \{d, 3 r+3\}}(\Omega), \quad$ and $f \in C^{\max \{d, 2 r+2\}}[0,1]$, then

$$
\tilde{z}_{n}^{M}-\varphi_{m}=\left(U(T(\varphi))+\frac{U\left(V_{1}(\varphi)\right)}{2}\right) h^{4 r}+O\left(h^{2 r} \max \left\{\tilde{h}^{d}, h^{2 r+2}\right\}\right) .
$$

Proof. Recall from (4.1) that

$$
\tilde{z}_{n}^{M}-\varphi_{m}=\mathscr{K}_{m}^{\prime}\left(\varphi_{m}\right)\left(z_{n}^{M}-\varphi_{m}\right)+O\left(\max \left\{\tilde{h}^{d}, h^{3 r}\right\}^{2}\right)
$$

Recall from (4.6) that

$$
\begin{aligned}
& \mathscr{K}_{m}^{\prime}\left(\varphi_{m}\right)\left(z_{n}^{M}-\varphi_{m}\right) \\
= & -L_{m} \mathscr{K}_{m}^{\prime}\left(\varphi_{m}\right)\left\{\mathscr{K}_{m}\left(\varphi_{m}\right)-\tilde{\mathscr{K}}_{n}^{M}\left(\varphi_{m}\right)\right\} \\
& +L_{m} \mathscr{K}_{m}^{\prime}\left(\varphi_{m}\right)\left\{\tilde{\mathscr{K}}_{n}^{M}\left(z_{n}^{M}\right)-\tilde{\mathscr{K}}_{n}^{M}\left(\varphi_{m}\right)-\left(\tilde{\mathscr{K}}_{n}^{M}\right)^{\prime}\left(\varphi_{m}\right)\left(z_{n}^{M}-\varphi_{m}\right)\right\} \\
& +L_{m} \mathscr{K}_{m}^{\prime}\left(\varphi_{m}\right)\left\{\left(\left(\tilde{\mathscr{K}}_{n}^{M}\right)^{\prime}\left(\varphi_{m}\right)-\mathscr{K}_{m}^{\prime}\left(\varphi_{m}\right)\right)\left(z_{n}^{M}-\varphi_{m}\right)\right\} .
\end{aligned}
$$

Thus, by Proposition 4.4, estimate (4.17) and Proposition 4.6, we obtain

$$
\mathscr{K}_{m}^{\prime}\left(\varphi_{m}\right)\left(z_{n}^{M}-\varphi_{m}\right)=\left(U(T(\varphi))+\frac{U\left(V_{1}(\varphi)\right)}{2}\right) h^{4 r}+O\left(h^{2 r} \max \left\{\tilde{h}^{d}, h^{2 r+2}\right\}\right)
$$

and the proof is complete. 
We can now apply one step of Richardson extrapolation and obtain approximations of $\varphi$ of higher order. Define

$$
z_{n}^{E}=\frac{2^{4 r} \tilde{z}_{2 n}^{M}-\tilde{z}_{n}^{M}}{2^{4 r}-1}
$$

Then we have the following result.

Corollary 1. Under the assumptions of Theorem 4.7 ,

$$
\left\|z_{n}^{E}-\varphi_{m}\right\|_{\infty}=O\left(h^{2 r} \max \left\{\tilde{h}^{d}, h^{2 r+2}\right\}\right)
$$

If we choose $\tilde{h}$ and $d$ such that $\tilde{h}^{d} \leq h^{2 r+2}$, then

$$
\left\|z_{n}^{E}-\varphi_{m}\right\|_{\infty}=O\left(h^{4 r+2}\right)
$$

\section{$5 \quad$ Numerical Results}

In this section we quote the results from Kulkarni-Nidhin [6] to illustrate the improvement of orders of convergence by the Richardson extrapolation obtained in (4.25).

Consider

$$
\varphi(s)-\int_{0}^{1} \frac{d s}{s+t+\varphi(t)}=f(s), \quad 0 \leq s \leq 1,
$$

where $f$ is so chosen that $\varphi(t)=\frac{1}{t+1}$ is a solution of (5.1).

We consider the following uniform partition of $[0,1]$ :

$$
0<\frac{1}{n}<\frac{2}{n}<\cdots<\frac{n}{n}=1
$$

For $r=1,2$, let $\mathscr{X}_{n}$ be the space of piecewise polynomials of degree $\leq r-1$ with respect to the partition (5.2). The collocation points are chosen to be $r$ Gauss points in each subinterval.

If $\mathscr{X}_{n}$ is the space of piecewise constant functions, then we choose the composite 2 point Gaussian quadrature with respect to the uniform partition of $[0,1]$ with 256 intervals. The computations are done for $n=2,4,8,16$ and 32 . Thus,

$$
r=1, d=4, \tilde{h}=2^{-8}, h \geq 2^{-5} \text { and hence } \tilde{h}^{d}=2^{-32} \leq 2^{-20} \leq h^{2 r+2}
$$

and the conditions of Theorem 4.7 are satisfied. 
If $\mathscr{X}_{n}$ is the space of piecewise linear functions, then we chose the composite 2 point Gaussian quadrature with respect to the uniform partition of $[0,1]$ with $n^{2}$ intervals. Thus,

$$
r=2, \tilde{h}=h^{2}, d=4 \text { and hence } \tilde{h}^{d}=h^{8} \leq h^{6}=h^{2 r+2}
$$

and the conditions of Theorem 4.7 are satisfied.

Table 4.4, Interpolation at Midpoints: $r=1$

\begin{tabular}{|c|ll|ll|ll|}
\hline$n$ & $\left\|\varphi-z_{n}^{M}\right\|_{\infty}$ & $\delta^{M}$ & $\left\|\varphi-\tilde{z}_{n}^{M}\right\|_{\infty}$ & $\delta^{I M}$ & $\left\|\varphi-z_{n}^{E}\right\|_{\infty}$ & $\delta^{E}$ \\
\hline 2 & $6.92 \times 10^{-3}$ & & $3.30 \times 10^{-4}$ & & & \\
4 & $1.02 \times 10^{-3}$ & 2.76 & $2.13 \times 10^{-5}$ & 3.95 & $7.31 \times 10^{-7}$ & \\
8 & $1.40 \times 10^{-4}$ & 2.87 & $1.34 \times 10^{-6}$ & 3.99 & $6.81 \times 10^{-9}$ & 6.75 \\
16 & $1.82 \times 10^{-5}$ & 2.94 & $8.37 \times 10^{-8}$ & 4.00 & $8.46 \times 10^{-11}$ & 6.33 \\
32 & $2.27 \times 10^{-6}$ & 3.00 & $5.23 \times 10^{-9}$ & 4.00 & $1.01 \times 10^{-12}$ & 6.39 \\
\hline
\end{tabular}

Table 4.6, Interpolation at Gauss 2 points: $r=2$

\begin{tabular}{|c|ll|ll|ll|}
\hline$n$ & $\left\|\varphi-z_{n}^{M}\right\|_{\infty}$ & $\delta^{M}$ & $\left\|\varphi-\tilde{z}_{n}^{M}\right\|_{\infty}$ & $\delta^{I M}$ & $\left\|\varphi-z_{n}^{E}\right\|_{\infty}$ & $\delta^{E}$ \\
\hline 2 & $5.06 \times 10^{-4}$ & & $6.47 \times 10^{-5}$ & & & \\
4 & $1.07 \times 10^{-5}$ & 5.56 & $2.09 \times 10^{-7}$ & 8.27 & $4.37 \times 10^{-8}$ & \\
8 & $1.85 \times 10^{-7}$ & 5.86 & $8.45 \times 10^{-10}$ & 7.95 & $2.67 \times 10^{-11}$ & 10.68 \\
16 & $3.07 \times 10^{-9}$ & 5.90 & $3.35 \times 10^{-12}$ & 7.98 & $4.73 \times 10^{-14}$ & 9.14 \\
32 & $4.74 \times 10^{-11}$ & 6.02 & $1.34 \times 10^{-14}$ & 7.96 & $2.11 \times 10^{-15}$ & 4.49 \\
\hline
\end{tabular}


The expected orders of convergence from (1.5), (1.7) and (4.24) are as follows:

Discrete Modified Projection Solution: $\delta^{M}=3 r$,

Discrete Iterated Modified Projection Solution: $\delta^{I M}=4 r$,

Extrapolated Solution: $\delta^{E}=4 r+2$.

It is clear from the above tables that the computed orders of convergence match well with the theoretical orders of convergence and that the extrapolated solution improves upon the discrete iterated modified projection solution.

\section{References}

[1] K. E. ATKINSON, The Numerical Solutions of Integral Equations of the Second Kind, Cambridge University Press, Cambridge, U.K., 1997.

[2] K. E. ATKINSON and J. FLORES, The discrete collocation method for nonlinear integral equations, IMA J. of Numerical Analysis, 13 (1993), 195 - 213.

[3] K. ATKINSON, I. GRAHAM and I. SLOAN, Piecewise continuous collocation for Integral Equations, SIAM J. of Numerical Analysis, 20 (1983), pp. 172-186.

[4] K. E. ATKINSON and F. A. POTRA, Projection and iterated projection methods for nonlinear integral equations, SIAM J. Numer. Anal., 24 (1987), 1352 - 1373.

[5] L. GRAMMONT, R. P. KULKARNI and P. VASCONCELOS, Modified projection and the iterated modified projection methods for nonlinear integral equations, J. Integral Equations Appl. Volume 25, (2013), 481-516.

[6] R. P. KULKARNI and T. J. NIDHIN, Asymptotic error analysis of projection and modified projection methods for non-linear integral equations, J. Integral Equations Appl., Volume 27 (2015), 67-101.

[7] R. P. KULKARNI and G. RAKSHIT, Discrete modified projection method for Urysohn integral equations with smooth kernels, Applied Numerical Mathematics, 126 (2018), 180-198. 